\title{
TE WHAI AU I TE TIRA HAERE
}

Hai whakapure i te tatau o tēnei pātaka korero a MAI Kohinga Reo-Rangahau 2018, $\bar{a}$-ipurangi ka tîkina atu te rārangi tuaiwa o te waiata tangi a tēnei kuia tohunga rawa ki te tito i ngā waiata o te rau tau 19 tuangahuru, a Mihikitekapua ō Ngāi Tūhoe, Ngāti Ruapani. Ko tēnei waiata āna e mōhio whānuitia ana ko Engari te titi e tangi haere ana (Black, 2014). Kai te whiti tuarua o tēnei waiata tēnei rārangi ātaahua, hohonu, rārangi matakite, ana whakamua Tè whai au $i$ te tira haere. Koia nei te ingoa, te taitara o tēnei pātaka korero a MAI Kohinga Reo-Rangahau 2018, $\bar{a}$-ipurangi Té whai au i te tira haere.

$\overline{\mathrm{E}}$ rua ngā whakamārama o tēnei rārangi ātaahua, hohonu Tè whai an i te tira haere...

Ko te whakamārama tuatahi ko te kupu nei tühono. Ahakoa he aha te kaupapa o to kaupapa reo-rangahau, ko te ahurewa nui o to rangahau ko te kupu nei tūhono ki te tangata, tūhono ki te tangata matatau hai āwhina i to kaupapa kia tipu, kia ora kia whakaaratia te mita, te hā o te kōrero. Kia pūare tonu ngā āhuatanga ki ngā kokona kāinga matatau o te tangata nei hai āwhina, hai whakawhānui, hai whakamāro, hai whakawhāiti, hai whakatipu kaha i tō kaupapa reo-rangahau kia ū, kia mau, kia hāngai. Ko te kupu nei tūhono ki waenga i a koe me taua tangata, kia kaua e whati! Whakamahia tō kaupapa reo-rangahau kia noho ko te kupu nei tūhono hai manaaki i te ahurewa nui, whakawhiti o te reo oro, te reo tuakiri, te mauri o te reo whakaora i te kairangahau kia takoto pai te tauira o tēnā kaupapa reo-rangahau, ō tēnā kaupapa reo-rangahau. Ko te āhua a te kupu nei tūhono he whakakotahi i te reo kōrero o te tangata matatau, me te reo kōrero o ngā whakaritenga tuhituhi hai whakatauira i te huarahi tuhituhi, whakawhāiti tahi i te kōrero o tō kaupapa reo-rangahau. Whāia aua āhuatanga e rua, te reo matatau o te tangata, me te reo matatau o te momo tuhituhi kia tūhono tonu hai āwhina, whakatauira i te kōrero Tè whai au i te tira haere... .

Ko te whakamārama tuarua o tēnei rārangi waiata Tè whai au i te tira haere e pēnei ana. Kai te takabi atu koe, te kairangahau $i$ te huarabi o tō kaupapa reo-rangahau. Ahakoa he aha tō kaupapa reo-rangahau kai te takahi koe te kairangahau, he huarabi hou, he huarahi mātāpono, he huarahi matakite hou katoa ēnei o te kaupapa nei te reo-rangahau. Nui atu ngā whakakitenga, ngā purapura nui, ngā huarabi o ngā puna mātauranga hou o Te Ao Māori, o Te Ao Tauiwi, Te Ao Ipurangi, Te Ao Taiao, Te Ao Tai Whetu, Te Ao Tai Whakanoho Kōrero, Te Ao Whakawhāiti kōrero ka kitea e te kairangahau. Koia tēnei te huarahi ka takahi e tō kaupapa reo-rangahau. Āta tātaritia tō takahi, tō hikoi ki roto i tō kaupapa reo-rangahau 
e tutuki ai te kaupapa Té whai an i te tira haere...

E pēnei ana te kōrero a te kuia nei a Mihikitekapua mo te rārangi waiata nei $T \bar{e}$ whai an i te tira haere ... me hohou tonu te rongo o te tühono me te huarahi takahi o tō kaupapa reo-rangahau kia kotahi tō rāua haere tahi, kia pūpū tonu kia mau te kotahitanga o te whakaaro. Ka kotahi ana rāua te tūhono me te huarahi takahi o tō kaupapa reo-rangahau, kai kona ka kitea te ora, te mauri, te mita, te hā o te tuakiri, te oro, te tiketike o tēnei rārangi ātaahua, hohonu kupu whakaritenga o te whare whakawhanaunga kōrero nei Té whai au i te tira haere...

\section{Kupu maioha ki a Ranginui Walker}

He manawa wera-haka tēnei i titoa e ahau e Taiarahia Black mo tēnei tangata a Ranginui Walker. He kanohi nui, hinengaro karawhiu o te auau o te kupu ki roto o Te Whare Wānanga o Tāmaki Makaurau, kā hia nei ngā tau. He ārero koi o te matamata o te hohonu, o te rākau nei te Kahikātoa. He mura rārauwhe mō te whakatakoto kaiā kōrero, he kawaka o te rerenga o te matamata koi, whakanoho kōrero. He puna kōrero e kore e mimiti. Ko te manawa-wera haka nei he mea whakaara ake i runga anō i te ātaahua, $i$ te hohonu o te whakarongo, o mātaki i te tangata nei i a Ranginui e kauhau ana. Whakapau kaha a Ranginui i tana ao mātauranga ki te tūhono, ki te takahi i te huarahi tika, pono kia tāua te Māori, ka mutu ka tūhono ia ki ngā mātauranga hohonu o Te Ao Māori. Koia tēnei manawa wera-haka Pōhēhē nei koe nō te korekore au te Māori.

\section{Pōhēhē nei koe no te korekore, no te pōhara au te Māori}

He whakarehu, he maimai aroha, he apakura, he poroporoaki, he manawa wera-haka tēnei mō Ranginui Walker. He ngārahu a Ranginui Walker o te toa kaitauā o te ao horapa i te kupu kōrero ā-waha, ā-tuhituhi, ā-tinana, ā-hinengaro, ā-pouaka whakaata, ā-reo irirangi, à-tuhi niupepa, ā-pukapuka, ā-tuhi ātikara ki te motu, ki te ao whānui auau ai! Ko wai o tātau o ngā kete kōrero, matapihi whakaaro o te motu kāre i takapore i ngā kupu hohonu, mārama, makariri o te ihi, o te taupatu o te whakaaro a tēnei toa o te kaiapo kupu o Te Whakatōhea; o Mākeo maunga, o Ōmarumutu whakawhiti rā, o Ngāti Rua toko manawa, o Te Whakatōhea mai i Tawhiti. Ko wai o tātau o tēnei motu kāre i tukia e te kupu hahani-wewete, tātari whakahiwā i te māramatanga i pakaru ai te kupu ki tana tuhituhi o te kawenata Ka Whawhai Tonu Mātou; Ka Whawhai Tonu Mātou ki Ōrakau, ki Te Tarata ka uru ki ngā toto o Ranginui Walker tēnei manu whititua o te riri. Ko wai o tātau kāre i tūnounou te māhunga i ngā whakaaro a tēnei toa whakahira o te whakaaro o Te Whakatōhea. Koia ka whakaingoatia e au tēnei whakarehu, maimai aroha, apakura, poroporoaki, manawa wera-haka o te kounga o te whakaaro: Pōhēhē nei koe no te korekore, no te pōhara au te Māori.

Huhua ngā kōrero a tēnei kārearea e maiangi mai nei ki te rangi, a tēnei kāhu koi tara whiti, a tēnei matuku o te hunga matatuhi. Koia ēnei manu Māori whakaara i te pō, whakawātea i te whakaaro kia mau te kupu kōrero. Mā wai atu hai tuhi ngā kōrero mo He Tipua, ko wai atu te tangata kai tēnei ao e whakaae ana a Ngāti Porou māna hai kato ngā kōrero mo He Tipua ka wetewete mai ki mua i a tātau katoa Māori mai, Pākehā mai. Ko wai atu te tangata hai toitū-takawaenga i ngā tukituki i waenga i te Māori me te Pākehā o aua tau o te pōhēhē o te tukituki whakahāwea o ngā tau 1970 haere ake nei, i hīkoi mai ai tātau te Māori. He kārearea, he kāhu, tēnei matuku, tēnei manu whakaara i te pō, whakaawatea i te whakaaro. Ko wai atu te tangata $\mathrm{i}$ kite atu i ngā taumata mātauranga a te Karauna, ka whakaputa i te kōrero ki te motu e kī pēnei ana "ko ènei taumata mātauranga a te Karauna kai te whakapōhara tonu i a tātan te Māori, ko te whakaeke ope tanā raupatu tuarua tēnei e haere mai nei” Inā ngā 
matakite a Ranginui Walker o Te Whakatōhea o Mākeo maunga, o Ōmarumutu whakawhiti rā, whakaara i te whakaaro, o Ngāti Rua toko manawa, toitū o Te Whakatōhea mai i Tawhiti.

Nā Mata Toa o te pukapuka, kohinga whakaaro a tana kaituhi a Paul Spoonley ka horahia o roto o te kāinga kōrero o Ranginui Walker e kitea ai he aha i kaha ai ia ki te wero ingā hinengaro wātea, hinengaro kūware o te motu, hinengaro ngaro, hinengaro pōhēhē, hinengaro o te hunga kāpō, ē kāpō tonu nei! Kāpō hinengaro, kāpō karu, kāpō whakaaro, kāpō korero, kāpō tangata. Mai ka pakaru, ka tūwhera tana tatau kōrero ki runga marae, wānanga ki hea rānei ko ana akoranga, mahi whakangungu ki Te Whare Wānanga o Tāmaki kīhai rawa e kitea he nohanga, he puta whakaaro, engari ka kitea he whakaaetanga, ahakoa pēhea. Ahakoa ki hea rānei kikī ana, maru ana, ana wānanga $\mathrm{i}$ te hunga hiakai ki ana kōrero, ka mutu ka weroa haeretia e ia ngā hinengaro mutunga kore, o te hunga whakarongo! He wānanga katoa ēnei i waiho iho a Ranginui ki a tātau katoa. I tipu mai rā ēnei whakaaro ōna ki te whakatika i te hē, i te whakatika i te kūware, i te whakatika kia takoto tika te kōrero ki ngā kokona o te hunga whakaara i te mārautanga o te mātauranga, ahakoa tēhea mātauranga e kōrerotia nei. Ko tāna kia whai mana te ao Māori ki roto i ngā puna whakatōtō o te tai àtea horonuku, o te tai āhuru hororangi, o te tai horo tiketike parikārangaranga o te tika, o te horo nuku kōkiri whakahuihui o te kōrero ki te wāhi è tika ana!

Eke atu ana a Ranginui ki tētahi kaupapa ka mōhio pai tātau he reo kōrero ka whakaoratia e ia, hai ruruku hohonu i te whakaaro. Ko te āhua o te kawe a Ranginui ko tana kite, rongo i ngā mahi tūkino o aua rau tau o te raupatu whakaparahako, whakapōhara. Ko ngā hua o aua tau tūkino i riro kē ki te hunga whai rawa, ka ngaro rā ngā tika, ka ngaro rā ngā pono, ka whai rawa kē atu ko ètahi atu. Ka whakakōkoi te hinengaro o Ranginui Walker o Te Whakatōhea mai Tawhiti. Ko tātau i whakawhiwhia, ko tātau i whakakākahutia, ko tātau o te motu i whakaoreoretia haere ake nei, e korikori tonu nei tātau!

Ēngari he taha ngahau anō tō Ranginui Walker he taha manaaki, he taha whare whakawhanaunga, he taha kaitiaki, he taha pūmau i runga i te tika me te pono i ngā wā katoa ki ana mahi me ana tamariki, mokopuna me tana hoa aroha a Deidre. I waimarie tātau i a Deidre nāna i tahutahu te ahi kōmau o te kāinga o te hunga whakatipu i a rāua tamariki, ka noho wātea te hinengaro mākohi, ka noho wātea mai te hinengaro manaaki o Deidre, ka wātea a Ranginui Walker ki te kura, ki te kura nui i a tātau o te motu. Ki te àta noho koe ki te taha i a Ranginui ka rongo tonu koe te kōingo o tana ngākau pūmau, tana ngākau manaaki, tana ngākau kaitiaki. $\mathrm{He}$ tangata o te ao whetukituki, puku mahi o te ao whakawhirinaki ki tana hinengaro, he tangata o te ngākaunui, pono me te hūmarie kia eke tātau te Māori, te tauira Māori kai roto i ngā here pūmau o te mātauranga ki te kounga o te hinengaro mākohi. Ko tāna, ko tā Ranginui Walker me noho, me whai i te māramatanga, i te pono, i te tūhono ki ngā whakawhitinga kōrero o tā te Māori e oioi ai tātau ki te awatea. Me mau ki te tikanga mātauranga, tikanga tīwhana i te kupu, tikanga whakaora, tikanga kawenga i ngā kaupapa, kauhau huhua, nunui e tai pari mai nei, e tai timu mai nei mai i ngā kokona o ngā ngaru tūātea o tana moana a Toi-kai-rākau, Toi te Huatahi, Toi Matua te Au nei Rangi, ko ia takere nuku, ko ia takere rangi, ko Ranginui e tū iho nei!

\section{Pōhēhē nei koe no te korekore, no te pōhara au te Māori}

Pōhēhē nei koe Pākēhā no te korekore au te Māori?

Pōhēhē nei koe Pākēhā no te pōhara au, o te hinengaro pōhara?

Hara mai ki taku pōhara Pākēhā, kia kainga koe e aku kupu!

E taku hinengaro pōhara!

$\overline{\mathrm{E}}$ aku kupu o te ao pōhara, taku hinengaro 
o te ao pōhara, kai kona taku kaha, tukituki mai nei, kai taku ao pōhara!

İ à ha hā!

Hokia ki te riri, te riri o ua whakarīiriri, te riri o ua whakaora!

Hokia ki te riha, te whakarihariha o te tukituki whakaaro;

Whakamakuru nei te whare kōrero o taku hinengaro

Whakamakuru nei te whare kōrero o taku ārero,

He whare wānanga tōna rite, he whare karapipiti taku titiro,

Ko au te Māori te kura ketekete, ko au te Māori te kura wānanga;

Nō hea ngā mate, ngā kōrero pōhara e hahani mai nei i āu i te Māori?

Nō hea ngā mate, ngā ture pōhara, ngā anuanu pōhara e kai mai nei i taku pōhara nui?

He kai mutunga kore ēnei, koia ka whakakōkoi au a Ranginui i taku hinengaro

İ ā hahā!

Au, au, aue hā!

Tēnā!

Koia ka kōkoi au a Ranginui i taku hahani kaha ki a Pākēhā

Kai oreore ana te hinengaro o Pākēhā i āu!

Kai oreore ana te hinengaro o te Māori i āu!

Kite ana koe Pākēhā i tōu pōhara i aku kupu;

Kite ana koe Pākēhā i te mārama o taku ao pōhara!

Kaitoa, hara mai anō ki taku ao pōhara!

Taku ao pōhara ki te motu

Kake ki ngā tihi tapu o te whakaaro, o taku ao pōhara

Rapua te pono o te kōrero, tirohia te whakaara mai o te mana motuhake

Te tākiritanga o taku ārero, oreore ana te motu!

Te tākiritanga o taku hinengaro pakaru ana te weherua whakaaro
Te tākiritanga o taku kanohi ka takapore te kūware i taku pōhara hohonu

Ē Pākēhā nōhea mai taku pōhara?

\section{Anei! I ā ha hā!}

Nō tai pari, nō tai timu, nō tai āki mai o ngā ngaru tukituki i taku -

Nō ngā kete e toru o te pikitanga o

Tānuiārangi te kaitiaki i ngā Wānanga

Wānanga o Tikitikiōrangi, Wānanga o

Te Toi Huarewa, Wānanga o Te Toi o

Ngā Rangi

Wānanga o Toi-kai-rākau, Wānanga o

Toi Te Huatahi, Wānanga o te Toi

Poutiriao

Wānanga o taku tapuae, Wānanga o te hōkai nuku, Wānanga o te hōkai o Ranginui!

Ka makuru te kupu, kā hora ki te motu, ki ngā tai ē whā, Tai Tokerau, Tai Rāwhiti

Tai Hauāuru, Tai Tonga ka ora mai a Kete

Tūātea o te kāhui karakia, tikanga tapu;

Ka ora mai a Kete Tūāuri o te wānanga tēnā a Whiro,

Whiro o te wero kia matāra

Whiro o te riri kia whakatikahia,

Whiro o te riri o ua whakarīiriri, te riri o ua whakaora!

Whakahoki i a tātau ki te tukituki whakaaro;

Ngā tihi tapu wānanga whakatipu, whakatipu nei i ngā reeanga i ō kupu, i ō whakaaro e Rangi!

Kā pai tō mahi, e tipu nei mātau i roto i ō kupu tukituki o te ātea whakakoi hinengaro!

Ka āra mai ko Kete Aronui, Toi te Kupu, Toi te Mana, Toi te Whenua!

Werohia ki te rākau matarua

Werohia ki te rākau mataora

Werohia ki te rākau a Tānenuiārangi o te Wānanga!

$\bar{A}$ ha hā!

Tēnā ia karawhiua mai anō o kupu e Pākēhā! 
Ki taku ao pōhara!

Pōhēhē nei koe no te korekore au te Māori?

Pōhēhē nei koe no te pōhara au o te hinengaro pōhara?

Hara mai ki taku pōhara Pākēhā, kia kainga koe e aku kupu

Aku kupu o te ao pōhara, kai kona taku kaha, taku nui, taku hohonu, kai taku ao pōhara!

$\overline{\mathrm{I}} \mathbf{a}$ ha hā!

\section{Kupu aroha}

E Ranginui kōrua ko tō hoa rangatira ā Deidre, ā kōrua tamariki me a kōrua mokopuna me ngā hūngaona katoa. Kua moe nei koe e Ranginui ki te pō, ēngari ko tō āhua kai roto katoa i ngā reeanga o ngā tau whitu tekau haere ake nei ki ēnei tau rua mano takahi atu nei ki te Moana o Toi-kai-Rākau, ki o kāinga kōrero ò Te Awahou, ki tō awa a Waiaua, ki Mākeo maunga whakawhiti kōrero.

Ko te kupu whakamutunga mo tēnei manawa wera-haka mo Ranginui Walker me tiki atu i ngā kupu mihi taurangi a Hēnare K. Ngata i tuhia e ia i Tūranganui-ā-kiwa te marama o Poutu-te-rangi te tau rua mano mā tahi. (Walker, 2001).

Ko Tā Hēnare Ngata te tama a Tā Apiranga Ngata. Inā rā ana kupu:

Nō reira, e Rangi, he mihi atu tēnei nā māua ko taku tuahine, nā màtau te whānau a Apirana, a Ngāti Porou whānui hoki ki a koe, nāu nei $i$ whakairo tēnei taonga whakahirahira HE TIPUA The life and times of Sir Apirana Ngata hei whakamaumahara ki tō mātau pāpā.

E moe e Ranginui Walker, tiwhatiwha te pō, tiwhatiwha te ao!

Te kupu whakaara o MAI Kobinga

Reo-Rangahau 2018, ā-ipurangi

\section{Kupu Whakamihi}

Kīhai rawa e riro mā te tangata kotahi hai kōrero, hai whakaara ngā kupu whakamihi kia koutou katoa i whāngai mai i a koutou kaupapa reo-rangahau, tuhituhi ki tēnei pātaka kohinga kōrero. Ko te whakaputanga tuatahi nui tēnei o MAI Kohinga Reo-Rangahau 2018 à-ipurangi. He whare whakatipu kaupapa reorangahau a koutou tuhituhi, whakaora i ngā mahi whakawhiti, whakaū o te kupu whakakitenga kia manawa nui tātau ki a tātau kaupapa reo-rangahau. Ka roa ahau, mātau katoa ngā kaiētita matua, ngā kaiwhakawāwā e whakawā ana i a koutou tuhinga reo-rangahau, e pānui ana, e waihanga ana i te takoto o te kupu, i te whakaara o te kaupapa, i te whakawhāiti, i te rarau mai ō ngā whakaaro mai i ngā kokona kāinga o te hinengaro o tēnā, ō tēnā o koutou. $\overline{\mathrm{E}}$, mea rawa ake ka tika kia mihi ki a koutou mahi tuhituhi reo-rangahau!

\section{Te kupu whakaara i a MAI Kohinga Reo-Rangahau 2018, à-ipurangi}

I tērā tau 2017 i te marama o Pipiri i tukua mai e Ngā Pae o Te Māramatanga, e ngā Ahorangi ē rua nei, ngā māreikura o te ritorito kōrero a Tracey McIntosh, Jacinta Ruru, me te kaiwhakarite kakama nei a Kiri West-McGruer kia mātau o Te Whare Wānanga o Awanuiārangi e whakatakoto mai ana he huarahi tūhono. Kāre i roa i muri mai ka rangona te reo pōwhiri o $\mathrm{Ngā}$ Pae o Te Māramatanga ki runga o Waipapa marae. Ko Ahorangi Waimarie Nikora i whakaeke mai me tana tira; Tè whai au ite tira haere. Nau mai e te Ahorangi māreikura Waimarie hai kāpehu hou mo Ngā Pae o Te Māramatanga haere ake nei.

Hoki ake nei au, he tono rangatira, he taumata kōrero tūhono tā Ngā Pae o Te Māramatanga kia ngāi mātau o Te Whare Wānanga o Awanuiārangi, a Ahorangi Taiarahia Black, Dr Agnes McFarland me Ahorangi Nathan Matthews. Kia kotahi te takahi kia whakaae mātau o Awanuiārangi hai kaiētita matua 
hou mō tēnei pātaka whakatipu kaupapa reoranghau o MAI Kohinga Reo-Rangahau 2018 $\bar{a}$-ipurangi. Me whakaae ka tika! Aua atu, he kuaha tēnei kua whakapuakitia mai nei mō à tātau kaupapa huhua reo-rangahau auaha kua whakaaratia mai nei e koutou, e ngā kaituhi whakaū kōrero ki roto i tō tātau reo tūhono hai whakaora i te àtaahua, i te hohonu, i te mana, i te hua o te reo tātari, reo wetewete, reo whakawhitiwhiti o ā koutou kaupapa reo-rangahau.

He ariā matua hohonu, ātaahua a koutou mahi te hunga kaituhi i a koutou kaupapa reo-rangahau e hira whakamua ai tēnei pātaka kōrero o MAI Kohinga Reo-Rangahau 2018 $\bar{a}$-ipurangi. Ki te hanga i te kupu, ki te hanga i te whakaaro o ngā kokona kāinga kōrero o te momo reo-rangahau kai tēnā, kai tēnā o koutou, kia riro mā te waka whakatere, whakaū o te ioio tühono o MAI Kohinga ReoRangahau 2018 a-ipurangi e kawe a koutou tuhituhi mātauranga ki ngā kāinga kōrero o te hinengaro manaaki, hinengaro tātari kōrero, hinengaro whakapuāwai kaupapa reo-rangahau. Koia nei te kounga haepapa matatau o a koutou mahi reo-rangahau hai whakatipu kaha i a koutou, i a tātau kaupapa reo-rangahau hai whāngai, hai tūhono i ēnei momo mātauranga ki ō tātau reo hāpori whakapiki kōrero i te kounga me te tohungatanga o te momo reo whakatinana i te reo whai mana o te rangahau.

Ko te kōmata taketake mo ngā kaituhi whakatinana reo-rangahau kua tātaitia mai ki roto i tēnei pātaka kōrero o MAI Kohinga Reo-Rangahau 2018 ā-ipurangi kai konei ō koutou whakaaro, tikanga, tūmanako, nawe, hitōria, kōrero tuku iho, matakite, kaupapa rangahau mātauranga taketake, tohutohu mātauranga hou e takoto nui atu nei ki roto i a koutou tuhituhi. E āhei ai ā koutou kaupapa reo-rangahau kia whai whakaaro ki ngā momo kete wānanga o te takahi o te huarahi whakatakoto, tūhono kōrero. E takoto nei ki roto i ā koutou kupu, koutou ngā kaituhi he reo whai ōranga, he reo papa tūhono whakaara i te mātauranga kia tūhono me te whānau, hapū me te iwi hai pūpū nui, hai au, ahu whakamua, tirotiro whānui kia puāwai ki Te Ao Hou reorangahau. Kai konei, kai roto i tāu tuhituhi ka tipu tahi mai te huhua o te whakaaro hou, auaha tūbono, te ngākau nui o tō tātau reorangahau matatau e pā kaha nei ki o tātau wawata kia hira te pono, te kounga o te reo mo a tātau mahi tuhituhi, rangahau, whakapukapuka ā-ipurangi, akoranga whakangungu, pae tātari, pae whakawhiti, pae whakawhāiti kōrero. Kā tika hoki tukua ō koutou whakaaro reo-rangahau kia puta hai pae kōrero, hai pae karanga kia ngāi tātau katoa, kia mau te kotahitanga o te whakaaro tūbono e takabia nei e koutou. Poipoiā kia pūmau te reo-rangahau hai reo tuku iho, hai reo whakaora i te kaupapa o reo-rangahau ki ngā kokona e kore nei e rangonatia ana.

Te rākau rangatira nei te Pohutukawa he tohu whakaora mo MAI Kohinga Reo-Rangahau 2018 à-ipurangi

Kai ngā whārangi o tēnei pātaka kōrero a MAI Kohinga Reo-Rangahau 2018 ā-ipurangi e whakaaratia ana, e whakakitea ai tātau ki ngā momo kaupapa reo-rangahau a tēnā, a tēnā ō koutou. Ēhara i te mea ko ēnei whakaritenga, whakakitenga o reo-rangahau i ahu noa mai i te kokona kotahi o te hinengaro. I āta noho tēnā hinengaro ki te whāngai i tēnā hinengaro, $\bar{a}$, kitea ana te ngākau tūmanako o te kaupapa. Me taku mōhio kīhai rawa i moea e koutou te pō, ao pō kia puta i a koe tāu nei kaupapa reorangahau akoranga.

Koia hoki te āhua o te rākau rangatira nei te Pohutukawa ahakoa ia tukitukia mai e ngā hau niwhaniwha, e ngā hau tūruki, e ngā hau tāhengihengi, e ngā karawhiu mai o ngā tai e whā. Ka tū tōnu tēnei rākau rangatira i runga i te roroku o te toka, o te ki tahi o te whakaaro ka mau tonu te Pohutukawa. He rākau rangatira ahurei, amorangi, toa kaipakanga tēnei rākau ki ngā taiao aporei whakatō i ana pakiaka ki runga, ki raro kia mau kia tūtakinga ki te rangi. Koia te āhua o te kairangahau reo-rangahau 
akoranga kia kaha ki te pakanga ki tō kaupapa, kia amorangi o whakaaro ki te rapu ki runga, ki raro o te pakiaka whakatipu kaha i tō kaupapa ātaahua, hohonu, whakatairanga tonu kia mau Té whai an i te tira haere...

He pērā te āhua i te rākau rangatira nei te Pohutukawa i te whaiorooro o te whare kōrero o Tānemāhuta, Tāne o te Wānanga. Ka tū rangatira mai te Pohutukawa i ngā tau ōnamata, tae noa mai ki a tātau nei i ēnei rā. Ā i ngā marama mai o Whiringa-ā-rangi, Whiringaà-nuku; mea rawa ake kua whakaatutia mai ōna pua ātaahua, pūwherowhero, e muramura ana tētahi pua ki tētahi pua i aua marama o Whiringa-ā-nuku, o Whiringa-ā-rangi tau atu ki a Hakihea. Mutunga mai o te maranga nui mai o te ātaahua. He tau, he tau te āhua o te pūwherowhero o te whakaaro o ōna pua. Ka muramura ana te ātaahua o tētahi pua, pua pūwherowhero ki tētahi pua koia tēnei kai te whāngai tahi tētahi pua, rāua i a rāua ki ngā mīere reka o te hinengaro matatau, àtaahua, o te mīere hohonu o rāua nei momo mātauranga. Kai tua noa atu tēnei mīere ātaahua, hohonu, oha i a tātau te tangata!

Ka kaha te pūwhero mai o ngā hua o te Pohutukawa kua kitea e rāua he tūhono e whakangungua ai tētahi ki tētahi ka tipu te tira whakaaro e kite nei tātau e muramura mai ana. Whāngai tonu tētahi pua i tētahi pua e piri tata ana, he tohu o te whakamataku o te ihi! Kua whakakitea, whakaaratia mai e te rākau rangatira nei e te Pohutukawa tana whakaoranga, reo ātaahua te reo ohomauri, reo whakapiri, reo pitaritari. Ka aurere te taiao me ōna hua i roto $i$ te ao o te ngākaunui, i te ao mutuwhenua, i te ao onematua. Ka whakawhiti te kōrero, ka whakawhiti te rā ka pua ngā whakaritenga o te rākau rangatira nei te Pohutukawa whakawāwā i te taiao oranga. Kai kona te roa noa atu o te mātauranga ki ō tātau katoa rā reo-rangahau. Inārā te kōrero; he reka ia nei te mātauranga mo te roa noa atu o ōu nei rā mo te hunga kairangahau, reo-rangahau haere ake nei ngā tau Tē whai au i te tira haere...

Kai whea mai, kai whea atu he whakatauira mā tātau o Ngā Pae o Te Māramatanga o MAI Kohinga Reo-Rangahau 2018 ä-ipurangi, o te ipurangi tūhono i ngā kōrero. Koia te kōrero mo te rākau rangatira nei e pua ai ōna hua pūwherowhero i te marama o Whiringa ā-rangi, Whiringa ā-nuku, ā, hai i a Hakihea ka puāwai te reo kōrero o te ātaahua o ngā pua o te rākau rangatira te Pohutukawa ki a tātau katoa huri i te motu, tangata mai, kararehe mai ki tai timu, ki tai pari, ki te tai whakaoreore whakaaro, tai whakaoreore hinengaro.

Ko ā koutou kaupapa reo-rangahau akoranga o MAI Kohinga Reo-Rangahau 2018 ā-ipurangi i takahi, i hikoi mai i te marama o Pipiri 2017, i roto i ā koutou mahi whakawhitiwhiti kōrero o aua marama tae rawa ki a Hakihea kua oti ngā whakawāwā a te hunga kaiwhakawā. Koia hoki te wā e hura ai te ātaahua o ngā pua pūwherohero o te rākau rangatira nei te Pohutukawa. Whāngai tonu tētahi pua i tētahi pua, whāngai tonu tētahi reo-rangahau akoranga i tētahi reorangahau akoranga, pua noa ngā whare kōrero e rua i te ātaahua, i te hohonu i te orokohanga o te whakaaro Tè whai an i te tira haere.

\section{Te Au Whakamua}

Kai te hāhaka, kai te tipu, kai te kaha te hunga whakaora, whakaara i tō tātau reo ki roto i ngā taumata hou whakatipu i te momo reorangahau ki roto i tēnei pātaka kōrero o MAI Kohinga Reo Rangahau 2018 ä-ipurangi. Ka tika rā kia pakari ake tātau ki roto i ngā mahi tuhinga reo-rangahau, he aha hoki i kore ai? He momo reo-rangahau tēnei kua whakatūria mai nei i ngā kokona kāinga, e ngā hinengaro kakama, kōkoi kia whakatipuria ngā kaupapa rangahau e whakaaratia mai ai e tēnā, e tēnā o tātau o roto i tēnei pukapuka. Ko te mahi hoki a te kaituhi reo-rangahau he para i te huarahi ki tētahi kaupapa e manakotia ana e te ngākau, e hira oruoru ai te hinengaro whakatipu kōrero. He tuatahi tēnei momo pātaka kōrero kua whakaaratia mai e Ngā Pae o Te Māramatanga kia au ai, kia poipoiā ki roto i te ipurangi. Ko te 
mahi rā hoki o tēnei pātaka kōrero he takahi, he tūhono, he whakaora, he rapu i te mauri, i te hā, i te tuakiri o à tātau kaupapa reo-rangahau hai whakatiketike i tō tātau reo ki ngā kokona kāinga o te kaupapa ātaahua nei, reo-rangahau ki ngā taumata hou kōtuku, hou paearu hou āniwaniwa.

Koia te kaupapa matua mā tātau katoa te hunga tātaki whakatipu kaupapa reo-rangahau he tūhono, he takahi i a tātau kaupapa reorangahau ki ngā kitenge hou, ki ngā kitenga hai au whakamua i a tātau katoa. Ko te whakaaturanga nui kia kitea mai āu nei tuhituhi, te āhua o tāu whakanoho kōrero, āu momo matakite e riro ai mā tāu momo reo-rangahau hai whakatipu, whakatinana i te kaupapa e whakaritea nei e te kairangahau reo. He kāinga kōrero nui te ao mātauranga tawhito, te ao mātauranga hou, he puna hohou i te rongo hou o te whakaaro. Ko te hinengaro kakama kai te kite, kai te whakakākahu i taua kaupapa e ora ai ngā momo mātauranga whāngai i ngā kokona o te hinengaro o ō tātau hāpori reo rangahau ā-whānau, ā-hapū, ā-iwi, ā-marae, ā-kainga. Ka tika tonu kia tipu mai tēnei pātaka kōrero a MAI Kohinga Reo-Rangahau 2018 ä-ipurangi.

\section{Ngā bua o te whakapūmautanga o ngā tau- mata reo-rangahau}

- Te wāhi e kōrerotia ana te reo me aro tonu tātau kia tipu kaha te momo reo-rangahau ki taua wāhi ki roto i a tātau kōrero;

- Kia tipu kaha te momo reo-rangahau ki waenga ngā whānau, hapū, me te marae, me te kāinga, me te iwi ahakoa he aha te kaupapa;

- Kia kawea te momo reo-rangahau ki ngā kokona kāinga hou o tēnei mea te kōrero, rapua he taumata hou à reo-rangahau, $\bar{a}$, kaua hoki e mutu te rapu i aua taumata hou hai kawe, hai whakaara i a tātau kaupapa reo-rangahau;

- Whakaaratia he rautaki reo-rangahau, hai aha? Hai whakanui ake i ngā mātāpono mahi tūhono, takahi huarahi momo reorangahau ahakoa he aha te kaupapa;

- Āta whakaritea ngā taumata, ki hea rānei kia kaha ai te tipu o te kauhau o te kaupapa reo-rangahau hai tautoko i ngā wāhi kaha te whakamahia o te reo;

- Tautokotia ngā hāpori reo-rangahau a te whānau, hapū me te iwi, me ngā marae kāinga, me ōna hononga whenua kia kitea, kia rongohia mai tata, mai tawhiti te hohonu, te matakite o te reo-rangahau;

- Tautokotia, whakaoratia, kōrerotia, kauhautia ngā hāpori reo-rangahau matakite katoa o Ahunga Āta Whakarite 2016-2020 aua kaupapa Rautaki Hōtaka Rangahau, Te Reo me Ngā Tikanga, Te au Whai Rawa, Te Tai Ao, Te Mauri Ora, Te Rautaki Whakawhitinga Mātauranga, Te Rautaki Mahitahi, Te Rautaki Whakapiki Āheinga me te Raukaha. Kia whakaputaia aua kaupapa ki roto i te reo-rangahau, $\bar{a}$, kia piri pono te tūhono, takabi tata Ki Ngā Hoa. (Ahunga Āta Whakarite Strategic Direction 2016-2020 pukapuka);

- Kia tātau te hunga kua māia ki te whakaara i te kaupapa reo-rangahau kia kaha tātau ki te whakatipu i te whakatipuranga hou kia pakari ai rātau ki roto i ngā mahi reo-rangahau ki ngā kokona kāinga o te kōrero;

- Kia mōhio pai tātau ko te kaupapa reo-rangahau pukenga, māna e hihiri ai te ngākau ki te kohikohi i ngā kōrero i waho atu i ngā mea e mohiotia nei e tātau. Tipu ana he taumata reo-rangahau hou, tipu ana te reo paetara ki taua kāinga kōrero o Te $\mathrm{Au}$ Whakamua Té whai an i te tira haere.

\section{Ngā bua o te reo-rangahau ka tipu mai he momo reo bou, be kupu bou}

- Tipu ana te kaupapa reo-rangahau, tipu ana te au mai o ngā papa kupu hou hāngai ki te kaupapa o te reo-rangahau;

- Koia a kaupapa reo-rangahau, he nui ngā 
rerenga kōrero hou, te au mai o te mita, o te hā o te kaituhi, te hanga mai o ngā kupu, me ngā momo rerenga kōrero kia rapua he kupu hou hai whakaora mai i taua momo reo-rangahau;

- Ko tēnei mahi he whakatipu he papa kupu hou mo te āhua o te reo-rangahau he mahi whakawhānui, he mahi whakaara i taua rangahau, he mahi whakatū, whakaniko o te kupu mo taua rangahau;

- Me whakawhanake mai tētahi papa kupu hou, me āta hanga he mātāpuna kāhui kupu hou; pukapuka hou, ka wetewete ka rapu i ngā whakamārama o aua kupu pērā i te wetewete, whakamārama o te pukapuka rangatira o HE PĀTAKA KUPU te kai a te rangatira (Te Taura Whiri i Te Reo Māori 2008);

- I ngā mahi rangahau ā-whānau, ā-hapū, à-iwi, ā-marae, ā-whenua, ā-taiao ka tipu mai hoki he papa kupu hou i te mea ko ia kaituhi ko tāna mahi he whakatipu mai anō i āna kupu, rerenga kōrero ake, whakataukī, pepeha hai whakaora mai i te papa kupu hāngai tonu ki ngā wawata hai whakaora nui i te reo o taua hāpori;

- Ko tēnei papa kupu hou reo-rangahau, māna e whakaara mai te reo tuakiri, te hā, te mita o te reo, te mauri o te reo, te hāngaitanga o te reo ki tēnā whakatipuranga, ki tēnā whakatipuranga pae whakawhiti kōrero;

- Ka whakaaratia e te papa kupu hou he reo whakaata, he reo toi, he reo-rangahau, he reo korikori kia maranga tātau, ā, mā te hunga rangahau hou kia tīmata mōata mai rātau ki te tūhono ki te takahi, ki te hikoi i ngā purapura ātaahua o tēnei kaupapa mutunga kore te reo-rangahau Tè whai au $i$ te tira haere...

\section{Kupu Whakamihi ki ngā Kaiwhakawā}

Koutou katoa ngā kaiwhakawāwā o ngā tuhinga reo-rangahau a tēnā kaituhi, a tēnā kaituhi koia nui te mihi atu ki a koutou katoa. He mahi nui tā te kaiwhakawāwā, ki te kaiwhakawā i ngā kōrero a tēnā kaituhi, a tēnā kaituhi; ki te wetewete, ki te waihanga, ki te āta whakaaro mehemea kai te eke te kupu me te kaupapa ki ngā taumata e tika ana. Nā, koia anō hoki ko tā koutou mahi ngā kaiwhakawā he puna rauhi mai mehe, he wāriu nui anō te kaupapa reo-rangahau a tēnā kaituhi, a tēna kaituhi hai whakatipu mātauranga hou, mātauranga hohonu e tipu ai te māhuri nui o taua kaupapa rangahau hai āwhina i te reo-rangahau o te hāpori. Ko te hiahia hoki o te hunga $\mathrm{i}$ whakawāwā i a koutou tuhituhi reo-rangahau e haere mai ai te wā ka noho koutou, me a koutou tuhituhi hai taumata whakawhāiti reo-rangahau kaupapa mo tāu matakite, i tāu kaupapa ki mua i te aroaro o te hunga whakatipu reo-rangahau. Ko te mahi a ngā kaiwhakawā he whakamana i ngā kupu taurangi i tuhia e koutou kia tūbono kia takahi tonu i te mātauranga pūmau tonu, kia tukua, kia tipu te kupu whakamārama, kia whakaaratia kia kitea te ngākau hihiko whakatakoto, whakaoreore kōrero!

\section{Ka pai te noho tahi mai te tūhono mai, te takahi mai o te reo-rangahau me te reo ā-ipurangi}

Ko te reo-rangahau me te reo-ipurangi he kohikohinga kōrero tūbono, takabi huarabi hou. Ka tīkina ngā rārangi kōrero mo tēnei pukapuka, pātaka kōrero o Te whai au te tira haere whakaakoranga, kaupapa whakangungu taumata kōrero i ngā huhua whakaheke kōrero, whakatōpū kaupapa ā-tuhituhi, ā-pānui, $\bar{a}$-whakaaro, $\bar{a}-w a h a, \bar{a}-w h a k a a t u r a n g a$, $\bar{a}$-mahi, $\bar{a}$-whakarongo, $\bar{a}$-wetewete $\mathrm{i}$ hea mai nei. Kai konei ka whakaara ake, ka rapua e tātau te kounga, te hohonu, te ātaahua, te rerekē, te hāngai, te whakawhāiti o te kupu kōrero me ōna whakaaro pākihi nui. Ka tirohia te māramatanga o ā tātau momo tuhituhi e noho hāngai ana ki a tātau whakaritenga kōrero kai roto i ō tātau whānau, hapū, iwi, 
marae-kāinga, whenua, taiao e mau ana, e pupuritia ana. Ko tēnei pātaka kōrero, momo reo kōrero, tuhinga kōrero he kōrero pūmau tonu hai ako i a tātau ki ngā momo mātauranga whakawhitiwhiti tuhituhi, me ōna whakaaro tūhono, e takahi atu ai tātau i te pua kōrero o te reo-rangahau tāpokopoko.

Haere ake nei, koia tēnei tō tātau waka hou, kāinga whakatere kōrero ko reo-rangahau, ko reo ā-ipurangi. Nō reira ko tā tātau whakaritenga o te reo-rangahau, o te reo à-ipurangi he pūpū tonu kia mai te tūhono, te whakakotahi, te hikoi whakakotahi mai i a tātau kōrero ki te wāhi kotahi hai toimata (taumata) whakaora, whakatinana i aua kōrero kia whitikitia ki ngā kokona kāinga o te kairangahau ki hea mai nei haere ai, e takahi ana.

Ka tika te rārangi kōrero a te kuia ahu whenua, tuhituhi nei a Mihikitekapua hai whakakapi i a tātau kōrero whakapuaki i tēnei whakaputanga tuatahi o MAI Kohinga ReoRangahau 2018 ā-ipurangi kia noho tahi mai rāua a reo-rangahau, a reo ā-ipurangi he mauri whakaū i te pai!

Tè whai au i te tira haere 